\title{
Hydrocolloidal Dressings Reduce Adverse Reactions Induced by Injection of Azarcytidine: A Clinical Observation Study
}

\author{
Fuxiang Luo ${ }^{1}$, Jiaxin Wang ${ }^{1}$, Yu Ding ${ }^{1}$, Yangshen $\mathrm{He}^{2}$, Chunli Wang ${ }^{1, *}$, Tiantian Xiao ${ }^{2, *}$ \\ ${ }^{1}$ Department of Hematology, First Affiliated Hospital, Jinan University, Guangzhou, China \\ ${ }^{2}$ Department of Medicine, Hospital of Integrated Traditional Chinese and Western Medicine, Guangzhou, China \\ Email address: \\ jnuluofx@163.com (Fuxiang Luo), merryveraforever@163.com (Jiaxin Wang),dy932426@163.com (Yu Ding), \\ twcl@jnu.edu.cn (Chunli Wang),dgheys@163.com (Yangshen He),dgxiaott@163.com (Tiantian Xiao) \\ *Corresponding author
}

\section{To cite this article:}

Fuxiang Luo, Jiaxin Wang, Yu Ding, Yangshen He, Chunli Wang, Tiantian Xiao. Hydrocolloidal Dressings Reduce Adverse Reactions Induced by Injection of Azarcytidine: A Clinical Observation Study. American Journal of Nursing Science. Vol. 9, No. 5, 2020 , pp. 316-319.

doi: 10.11648/j.ajns.20200905.12

Received: August 3, 2020; Accepted: August 14, 2020; Published: August 25, 2020

\begin{abstract}
To investigate the effect of hydrocolloid dressings on reducing complications associated with azarcytidine injection. Methods: Twenty patients with myelodysplastic syndrome were included in the self-controlled study. The group settings were as follows: Experimental group A: Hydrocolloidal dressing was used alone after injection. Experimental group B: 50\% magnesium sulfate topical. Experimental group C: blank control group. Pain, anxiety and depression scores, and complications of subcutaneous ecchymosis, subcutaneous induration, subcutaneous swelling and skin erythema were compared in each group. Results: Both the hydrocolloid dressings and 50\% magnesium sulfate external application can reduce the local pain and reduce the occurrence of adverse emotions (A vs. $\mathrm{C}, \mathrm{P}=0.000, \mathrm{P}=0.000, \mathrm{P}=0.000$; $\mathrm{B}$ vs. $\mathrm{C}, \mathrm{P}=0.000, \mathrm{P}=0.092$, $\mathrm{P}=0.044$ ). Hydrocolloid dressings can effectively reduce the occurrence of skin ecchymosis, subcutaneous scleroma, subcutaneous swelling and skin erythema $\left.\left(\mathrm{X}^{2}=8.12, \mathrm{X}^{2}=8.12, \mathrm{X}^{2}=6.667, \mathrm{X}^{2}=3.956\right) . \mathrm{P}<0.01, \mathrm{P}<0.01, \mathrm{P}<0.022, \mathrm{P}<0.047\right)$. Conclusion: The external application of hydrocolloid dressing can significantly reduce the occurrence of skin related complications and patients' adverse emotions after azarcytidine injection, with definite curative effect and strong application. It is an efficient and convenient nursing method.
\end{abstract}

Keywords: Hydrocolloid Dressings, Azarcytidine, Complications

\section{Introduction}

Azacytidine is a new type of demethylation drug, which is one of the main drugs in the treatment of refractory and recurrent myelodysplastic syndrome (MDS) [1,2]. The efficacy of the drug in the treatment of MDS was evaluated after at least 4 courses of treatment. The common complications of subcutaneous injection of azacytidine include local pain, subcutaneous hemorrhage, subcutaneous hematoma, subcutaneous induration, skin necrosis, anxiety and depression, etc [3, 4]. The incidence of injection-related complications is very high. The occurrence of injection-related complications is closely related to whether patients can complete the standard course of treatment and the effect of treatment. How to use nursing drugs to reduce injection-related complications caused by subcutaneous injection of azacitidine and the occurrence of unhealthy emotions in patients is an important topic of current hematology specialist nursing care and research. In recent years, hydrocolloid dressings have been reported to be effective in the prevention and treatment of phlebitis, subcutaneous hemorrhage, pressure sore nursing and local skin infection [5-7]. Therefore, by observing the efficacy of hydrocolloid dressings in reducing complications associated with azacarbine injection, this study explored a simple, effective and easy to promote clinical nursing program.

\section{Information and Method}

\subsection{General Information}

20 patients treated by subcutaneous injection of 
azacytidine in our hospital from January 2017 to September 2019 were selected as research objects. The range of body fat, peripheral nerve sensory evaluation and mental cognitive status were normal and no skin edema, rash and scar. There were 9 males and 11 females, ranging in age from 45 to 64 years old. All patients signed informed consents, and this study met the requirements of the Declaration of Helsinki [8].

\subsection{Method}

\subsubsection{Process}

The azacitidine was redissolved with $4 \mathrm{~mL}$ sterile water for injection to obtain a $25 \mathrm{mg} / \mathrm{mL}$ homogeneous suspension, and equally divided into two syringes $(2 \mathrm{~mL} /$ bottle). The syringe is placed between the palm of the hand and rolled vigorously before injection,. Two syringes were injected into two different sites. Each injection is rotated to the injection site (thigh, abdomen or upper arm). The new injection site is at least $2.5 \mathrm{~cm}$ away from the old injection site. The patient took the supine position, wiped and disinfected the skin. The injector pinched the skin within the injection range for $5-6$ $\mathrm{cm}$ with the left thumb and index finger, to form a fold, and the right hand using holding pen-style to vertically needle at the highest point of the fold; after drawing back no blood, the injection drug was injected quickly and the needle was pulled out to continue to lift and pinch the skin $2 \mathrm{~min}$ and relax the skinfold. Use self - control methods.

\subsubsection{Group}

Group A: The patients were treated with hydrocolloid dressings after injection with following methods: $0.9 \%$ sodium chloride solution was used to clean the affected area, hydrocolloid dressings were used to cover the area with a diameter larger than $2 \mathrm{~cm}$, dressing was changed once a day for 7 consecutive days (the first course of treatment d1-7); In case of moisture or pollution should be replaced in time. Group B: Two layers of sterile gauze were soaked with $50 \%$ magnesium sulfate solution, with no water dripping, and wet applied to the affected area. Cover with plastic wrap for 30 min each time, 3 times a day, for 7 consecutive days (the second course of treatment d1-7). Group C: No treatment after injection (the third course of treatment d1-7).

\subsection{Evaluation}

\subsubsection{Local Pain}

20 minutes after injection, the patients were evaluated by digital pain rating (NRS) before and after nursing care. The pain was 10 points from no pain to severe pain. The patients evaluated themselves according to their actual conditions.

\subsubsection{Skin Ecchymosis}

Skin ecchymosis occurs or not after 12 hours of injection [9].

\subsubsection{Subcutaneous Induration}

Induration can be touched and pressure increases the pain.

\subsubsection{Regularly Observe Whether Swelling and Skin Erythema Occur After Injection}

The Hamilton Depression Scale (HAMD) score and Hamilton Anxiety Scale (HAMA) score were used to evaluate the emotional state before and after nursing treatment [13]. HAMD score for 17 items: total score $<7$ points: normal; total score from 7 to 17 : possible depression; total score from 17 to 24 : depression defined; total score: $>24$ : severe depression. HAMA score in 14 items: total score $<7$ : normal; total score from 7 to 17 : possible anxiety disorders; total score from 17 to 24 : confirmed anxiety; total score $>24$ : severe anxiety.

\subsection{Statistical Methods}

We performed statistical analysis using SPSS 20.0 statistical software. Chi-square test was used for counting data, the measurement data HAMD, HAMA and NRS scores are expressed as $(\mathrm{X} \pm \mathrm{s})$, and two independent sample t-tests were performed. $\mathrm{P}<0.05$ was considered statistically significant.

\section{Results}

\subsection{Comparison of Pain, Anxiety and Depression Scores Before and After Treatment}

The scores of NRS, HAMD and HAMA in hydrocolloid dressing group A and 50\% magnesium sulfate group B were significantly lower than those in control group $\mathrm{C}$ (A vs. C, $\mathrm{P}=0.000, \mathrm{P}=0.000, \mathrm{P}=0.000 ; \mathrm{B}$ vs. $\mathrm{C}, \mathrm{P}=0.000, \mathrm{P}=0.092$, $\mathrm{P}=0.044$ ), Hydrocolloid dressings and 50\% magnesium sulfate external applications after azacitidine injection can both relieve the pain caused by subcutaneous injection and reduce the occurrence of anxiety and depression to some extent. In addition, the effect of hydrocolloid dressing group was more significant than that of magnesium sulfate group (A vs. $\mathrm{B}, \mathrm{P}=0.008, \mathrm{P}=0.006, \mathrm{P}=0.035$ ).
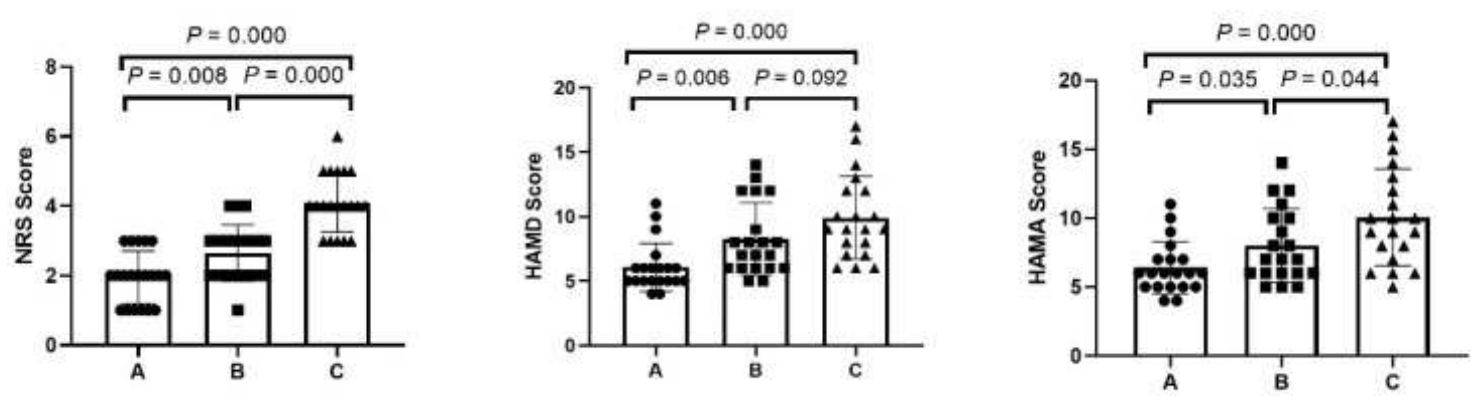

Figure 1. Comparison of HAMD, HAMA and NRS scores among 3 groups. 
Table 1. Comparison of NRS, HAMD and HAMA scores among three group (score, $\bar{x}+s$ ).

\begin{tabular}{llll}
\hline Group & NRS score & HAMD score & HAMA score \\
\hline A & $1.95 \pm 0.759$ & $6.05 \pm 1.88$ & $6.40 \pm 1.87$ \\
B & $2.65 \pm 0.813$ & $8.25 \pm 2.81$ & $8.00 \pm 2.67$ \\
C & $4.10 \pm 0.853$ & $9.90 \pm 3.21$ & $10.05 \pm 3.50$ \\
\hline
\end{tabular}

A hydrocolloid dressing group; B 50\% magnesium sulfate group; C control group.

\subsection{Comparison of Other Complications}

The results of chi-square analysis showed that the use of hydrocolloid dressing after drug injection could effectively reduce the incidence of skin ecchymosis, subcutaneous induration, subcutaneous swelling and skin erythema.
$\left(X^{2}=8.12, \quad X^{2}=8.12, \quad X^{2}=6.667, \quad X^{2}=3.956 ; \quad P<0.01, \quad P<0.01\right.$, $\mathrm{P}<0.022, \mathrm{P}<0.047$ ) (Table 2; But the $50 \%$ magnesium sulfate external application is not effective in reducing the incidence of the above complications. $(\mathrm{P}<0.191, \mathrm{P}<0.111, \mathrm{P}<0.343$, $\mathrm{P}<0.333$ ) (Table 3).

Table 2. Comparison of injection-related complications between hydrocolloid dressing group and control group (Frequency).

\begin{tabular}{lllll}
\hline Group & subcutaneous ecchymosis & subcutaneous induration & subcutaneous swelling & skin erythema \\
\hline A & 6 & 5 & 4 & 4 \\
C & 15 & 14 & 12 & 10 \\
$\mathrm{X}^{2}$ & 8.12 & 8.12 & 6.667 & 3.956 \\
P & 0.01 & 0.01 & 0.022 & 0.047 \\
\hline
\end{tabular}

A: hydrocolloid dressing group; C: control group.

Table 3. Comparison of injection-related complications between 50\% magnesium sulfate group and control group (Frequency).

\begin{tabular}{lllll}
\hline Group & subcutaneous ecchymosis & subcutaneous induration & subcutaneous swelling & skin erythema \\
\hline $\mathrm{B}$ & 10 & 8 & 8 & 6 \\
$\mathrm{C}$ & 15 & 14 & 12 & 10 \\
$\mathrm{X}^{2}$ & 2.667 & 3.636 & 1.000 & 1.667 \\
$\mathrm{P}$ & 0.191 & 0.111 & 0.343 & 0.333 \\
\hline
\end{tabular}

B 50\% magnesium sulfate group; C control group.

\section{Discussion}

Azacitidine is a new demethylated drug. At present, it is mainly used in refractory and recurrent MDS patients with poor efficacy of decitabine and in elderly patients with AML who can not tolerate chemotherapy and unconditional Hematopoietic stem cell transplantation. Use the drug to treat MDS for at least 4 courses of treatment. The common complications of azacytidine subcutaneous injection include local pain, subcutaneous hemorrhage, subcutaneous hematoma, subcutaneous induration, skin necrosis, anxiety and depression, etc $[3,4]$. The frequency of adverse reactions caused by injection of cytosine azacarbine is high. The occurrence of injection-related complications is closely related to whether the patients can complete the standard course of treatment and the therapeutic effect. At present, the research of using clinical nursing drugs to prevent azacytidine-induced injection-related complications and reduce negative emotions is still less. The ideal dressing should not only keep the skin around the wound dry, but also keep the bottom of the wound moist, absorb the exudate, and promote anti-inflammation to avoid the invasion of bacteria.

Hydrocolloid dressing is a new type of dressing commonly used in clinic. Mainly composed of sodium carboxymethyl cellulose, hydrocolloid dressing has good viscosity and airtightness so that form a closed room on the skin surface. The closed healing environment can promote the proliferation of microvessels and the formation of granulation tissue, thus accelerating the wound healing, and at the same time, the formation of hypoxic tension in the local area can stimulate the release of macrophages and Interleukin, and accelerate the local blood circulation, it speeds up the inflammation, relieve the pain $[10,11]$. At present, a large number of studies have shown that hydrocolloid dressings play a significant role in the prevention and treatment of phlebitis, ecchymosis, decubitus, and skin infection caused by indwelling catheter [5-7]. In this experiment, we observed the curative effect of 20 MDS patients who used hydrocolloid dressings to prevent azacitidine injection-related complications. The results showed that the hydrocolloid dressings reduced the degree of pain at the injection site, the complication rate of subcutaneous Induration, swelling and ecchymosis decreased significantly. The 50\% magnesium sulfate wet dressing can also reduce swelling and relieve pain by using its hypertonic properties. However, the dressing needs to be changed frequently, and it is affected by the environmental temperature and has poor compliance. In addition, our results show that magnesium sulfate external application is less effective than hydrocolloid dressing in reducing the incidence of skin ecchymosis, subcutaneous induration, subcutaneous swelling and skin erythema after azacytidine injection, which are consistent with some studies [12, 14, 15].

Some studies have shown that the hydrocolloid dressing's 
surface temperature is low, which not only cool and analgesic but also relieve inflammatory reaction rapidly. Strong therapeutic effect on relieving topical pain and less affected by ambient temperature and humidity, it greatly increases the comfort and compliance of patients. The above is also an important reason why hydrocolloid is widely used in clinical nursing to prevent and treat post-transfusion phlebitis. In addition, our research also found that the application of hydrocolloids can significantly reduce the occurrence of anxiety and depression in patients. A large number of studies have shown that repeated and continuous painful stimulation is one of the important causes of anxiety and depression in patients. A large number of studies have shown that repeated persistent pain stimulation is one of the major causes of anxiety and depression in patients [13]. In this study, hydrocolloid dressings reduce the pain of repeated subcutaneous injections, increase physical comfort, decrease injection complications. The above may be associated with the reduction of negative feelings in the experimental group and make more convenient to the patient completing the follow-up long time, the multi-course treatment.

\section{Conclusions}

To sum up, the hydrocolloid dressing can obviously reduce the skin-related complications and patients' negative feelings after azacytidine injection. The curative effect is both exactly and highly applicable, which greatly reduces the nurses' work burden and improves the patients' compliance. It is an efficient and convenient nursing method and worth popularizing in clinic.

\section{Acknowledgements}

Author Jiaxin Wang and Fuxiang Luo are the co-first authors of this paper in no particular order. Due to they have made equally important contributions to the design, experiment, data collation and writing of this paper.

\section{References}

[1] Montalban-Bravo G, Garcia-Manero G: Myelodysplastic syndromes: 2018 update on diagnosis, risk-stratification and management. American journal of hematology 2018, 93 (1): 129-147.

[2] Vigil CE, Martin-Santos T, Garcia-Manero G. Safety and efficacy of azacitidine in myelodysplastic syndromes. Drug Des Devel Ther. 2010, 4: 221-229.

[3] Shimoda-Komatsu Y, Mizukawa Y, Takayama N, Ohyama M. Cutaneous adverse events induced by azacitidine in myelodysplastic syndrome patients: Case reports and a lesson from published work review. J Dermatol. 2020; 47 (4): 363-368.

[4] Roy C, Adam JP, Morin F, Lemieux-Blanchard É, Doucet S, Friedmann D, Belisle A, Charpentier D: Azacitidine-induced pyoderma gangrenosum at injection sites in a patient with myelodysplastic syndrome. Current oncology (Toronto, Ont) 2018, 25 (1): e103-e105.

[5] Jurić Vukelić D, Jurić J: Hydrocolloid Dressing Application in the Treatment of Chronic Wounds and Relation to Quality of Life. Acta clinica Croatica 2017, 56 (3): 544-549.

[6] Cortés OL, Salazar-Beltrán LD, Rojas-Castañeda YA, Alvarado-Muriel A, Serna-Restrepo A, Grinspun D: Use of Hydrocolloid Dressings in Preventing Pressure Ulcers in High-risk Patients: a Retrospective Cohort. Investigacion y educacion en enfermeria 2018, 36 (1): e11.

[7] Bishopp A, Oakes A, Antoine-Pitterson P, Chakraborty B, Comer D, Mukherjee R. The Preventative Effect of Hydrocolloid Dressings on Nasal Bridge Pressure Ulceration in Acute Non-Invasive Ventilation. Ulster Med J. 2019; 88 (1): 17-20.

[8] World Medical Association Declaration of Helsinki: ethical principles for medical research involving human subjects. Jama 2013, 310 (20): 2191-2194.

[9] Mengiardi S, Tsakiris DA, Lampert ML, Hersberger KE: Drug use problems with self-injected low-molecular-weight heparins in primary care. European journal of clinical pharmacology 2011, 67 (2): 109-120.

[10] Zhang X, Sun D, Jiang GC: Comparative efficacy of nine different dressings in healing diabetic foot ulcer: A Bayesian network analysis. Journal of diabetes 2019, 11 (6): 418-426.

[11] Chamorro AM, Vidal Thomas MC, Mieras AS, Leiva A, Martínez MP, Hernández Yeste MMS: Multicenter randomized controlled trial comparing the effectiveness and safety of hydrocellular and hydrocolloid dressings for treatment of category II pressure ulcers in patients at primary and long-term care institutions. International journal of nursing studies 2019, 94: 179-185.

[12] Zheng GH, Yang L, Chen HY, Chu JF, Mei L: Aloe vera for prevention and treatment of infusion phlebitis. The Cochrane database of systematic reviews 2014, 2014 (6): Cd009162.

[13] Zis P, Daskalaki A, Bountouni I, Sykioti P, Varrassi G, Paladini A: Depression and chronic pain in the elderly: links and management challenges. Clinical interventions in aging 2017, 12: 709-720.

[14] Francesko A, Petkova P, Tzanov T: Hydrogel Dressings for Advanced Wound Management. Curr Med Chem. 2018; 25 (41): 5782-5797.

[15] Antonio Francesko, Petya Petkova and Tzanko Tzanov: Hydrogel Dressings for Advanced Wound Management. Current Medicinal Chemistry (2018) 25: 5782. 